\title{
Sub-microradian Surface Slope Metrology with the ALS Developmental Long Trace Profiler
}

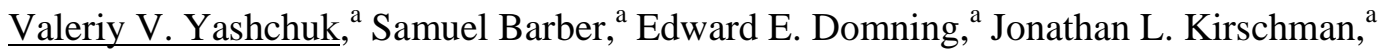

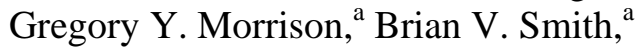 \\ ${ }^{a}$ Lawrence Berkeley National Laboratory, One Cyclotron Road, \\ Berkeley, California 94720, USA \\ Frank Siewert, ${ }^{\text {b }}$ Thomas Zeschke, \\ ${ }^{\mathrm{b}}$ Helmholtz Zentrum Berlin für Materialien und Energie, Elektronenspeicherring BESSY-II, Albert- \\ Einstein-Str. 15, 12489 Berlin, Germany \\ Ralf Geckeler, ${ }^{\mathrm{c}}$ Andreas Just ${ }^{\mathrm{c}}$ \\ ${ }^{\mathrm{c}}$ Physikalische-Technische Bundesanstalt (PTB), Bundesallee 100, 38116 \\ Braunschweig, Germany
}

\section{Introduction}

Development of X-ray optics for 3rd and 4th generation X-ray light sources with a level of surface slope precision of 0.1-0.2 $\mu$ rad requires the development of adequate fabrication technologies and dedicated metrology instrumentation and methods. Currently, the best performance of surface slope measurement has been achieved with the NOM (Nanometer Optical Component Measuring Machine) slope profiler at BESSY (Germany) [1] and the ESAD (Extended Shear Angle Difference) profiler at the PTB (Germany) [2]. Both instruments are based on electronic autocollimators (AC) precisely calibrated for the specific application [3] with small apertures of 2.5 - $5 \mathrm{~mm}$ in diameter.

In the present work, we describe the design, initial alignment and calibration procedures, the instrumental control and data acquisition system, as well as the measurement performance of the Developmental Long Trace Profiler (DLTP) slope measuring instrument recently brought into operation at the Advanced Light Source (ALS) Optical Metrology Laboratory (OML). Similar to the NOM and ESAD, the DLTP is based on a precisely calibrated autocollimator. However, this is a reasonably low budget instrument used at the ALS OML for the development and testing of new measuring techniques and methods. Some of the developed methods have been implemented into the ALS LTP-II (slope measuring long trace profiler [4]) which was recently upgraded and has demonstrated a capability for $0.25 \mu$ rad surface metrology [5].

Performance of the DLTP was verified via a number of measurements with high quality reference mirrors. A comparison with the corresponding results obtained with the world's best slope measuring instrument, the BESSY NOM, proves the accuracy of the DLTP measurements on the level of 0.1-0.2 $\mu$ rad depending on the curvature of a surface under test. The directions of future work to develop a surface slope measuring profiler with nano-radian performance are also discussed.

\section{DLTP Design}

Figure 1 illustrates the DLTP design.

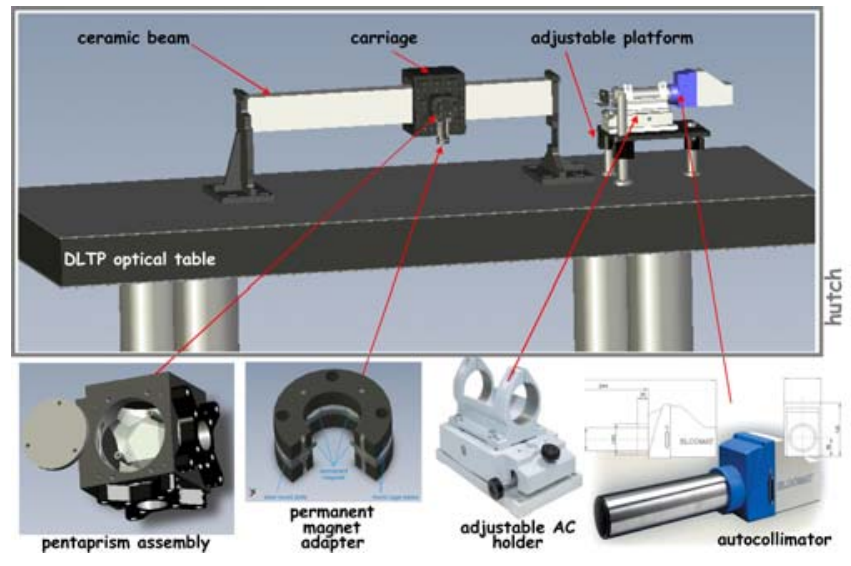

Figure 1: The main DLPT parts are an autocollimator "Elcomat 3000 special" (Moeller Wedel Optical [6]) calibrated at the PTB and a movable pentaprism mounted on the air-bearing carriage translated along a ceramic beam with a Nanomotion motor. The adjustable AC holder, pentaprism kinematic stage, and specially designed permanent magnet adapter for an adjustable diaphragm are used for precision alignment of the DLTP parts to ensure minimal systematic error of the slope measurement [7]. The surface under test (SUT) must be oriented face-up. 


\section{DLTP Measurement Performance}

Performance of the DLTP was verified via a number of measurements with high quality reference mirrors. As an example, Fig. 2 presents cross-check measurements with a super high quality flat mirror performed with the DLTP and the upgraded ALS LTP-II. Similar performance has been also demonstrated by measuring significantly more curved reference optics with radii of curvature of 1280 meters and 15 meters and comparing the results with the data obtained with the BESSY NOM.
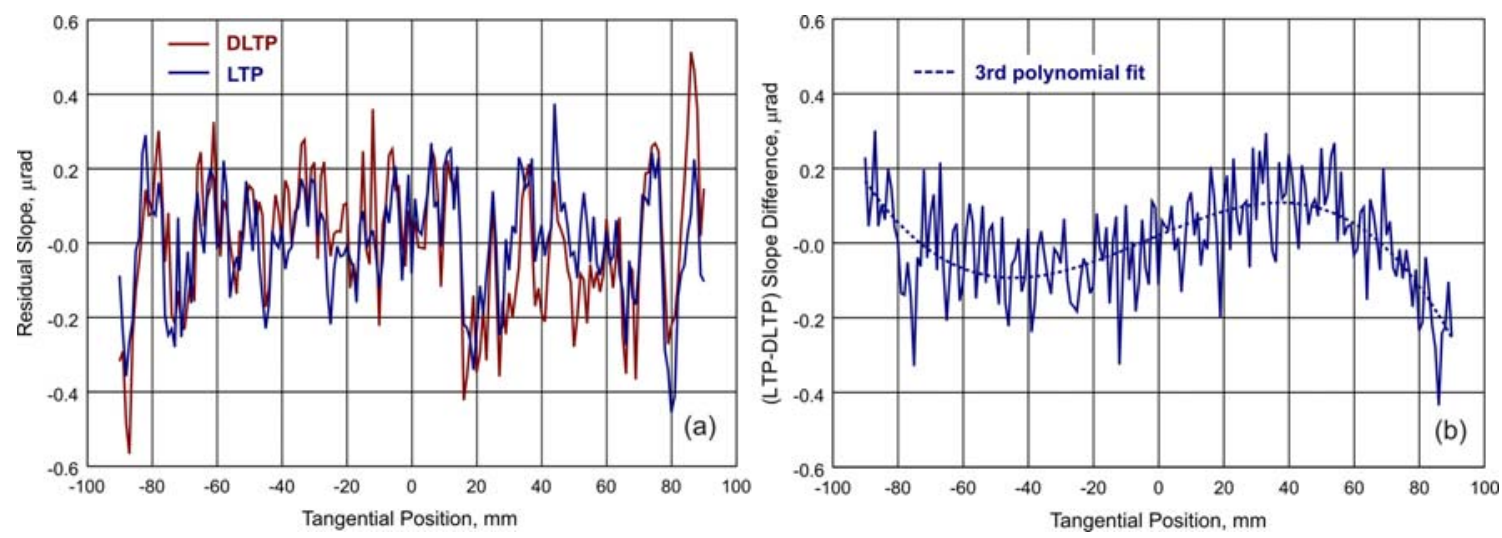

Figure 2: (a) Metrology of a super high quality flat mirror performed with the DLTP (the red line) and the ALS LTP-II (the blue trace). The slope variation is $0.18 \mu \mathrm{rad}$ (rms) and $0.15 \mu \mathrm{rad}$ (rms) as measured with the DLTP and the LTP, respectively. (b) The difference of the measurements in (a). The rms variation of the difference is $0.13 \mu \mathrm{rad}$. A systematic discrepancy of the instruments that is fitted with the $3^{\text {rd }}$ order polynomial (the dashed line) is about $0.2 \mu$ rad (peak-to-valley). The residual random noise of the measurements is about $0.07 \mu \mathrm{rad}$ (rms). The measurements were made with the lab air conditioner switched off and using the optimal scanning strategy discussed in Ref. [8].

In this article, we discuss the experimental precautions and finesses that allow us to minimize the errors due to the systematic effects and instrumental drift [8].

\section{Future Developments}

The performance of the current DLTP set-up is limited by a number of systematic errors. The systematic error due to the finite quality of the bulk pentaprism would be significantly eliminated by using a pentaprism made of precisely adjusted super flat mirrors. The limitation related to the AC calibration at a fixed distance between the AC and the SUT, would be overcome with a sophisticated in-situ calibration of the profiler with a method proposed in Ref. [9]. A calibration system based on that method is under development by the collaboration between the ALS, BESSY, and the PTB.

In order to fully realize the advantages of suppression of the instrumental drift error with the optimal scanning strategies derived in Ref. [8], a Huber-stage-based rotation system for automatic flipping the SUT is being developed.

\section{Acknowledgements}

The authors are grateful to Wayne McKinney and Howard Padmore for useful discussions and to Tom Tonnessen, Jim Mentz, Amparo Rommeveaux, Muriel Thomasset, and all Round Robin collaborators for providing the reference mirrors and the data of the Round Robin measurements. The Advanced Light Source is supported by the Director, Office of Science, Office of Basic Energy Sciences, Material Science Division, of the U.S. Department of Energy under Contract No. DE-AC0205CH11231 at Lawrence Berkeley National Laboratory.

\section{Disclaimer}

Certain commercial equipment, instruments, or materials are identified in this document. Such identification does not imply recommendation or endorsement by the US Department of Energy, LBNL, or ALS, nor does it imply that the products identified are necessarily the best available for the purpose. 


\section{References}

[1] F. Siewert, T. Noll, T. Schlegel, T. Zeschke, and H. Lammert, "The Nanometer Optical Component Measuring machine: a new Sub-nm Topography Measuring Device for X-ray Optics at BESSY,” Proc. AIP 705, 847-850 (2004).

[2] R.D. Geckeler, “ESAD Shearing Deflectometry: Potentials for Synchrotron Beamline Metrology,” Proc. SPIE 6317, 63171H/1-12 (2006).

[3] A. Just, M. Krause, R. Probst, and R. Wittekopf, "Calibration of high-resolution electronic autocollimators against an angle comparator,” Metrologia 40, 288-294 (2003).

[4] P. Takacs, S. N. Qian and J. Colbert, "Design of a long trace surface profiler," Proc. SPIE 749, 59-64 (1987). [5] J. L. Kirschman, E. E. Domning, W. R. McKinney, G. Y. Morrison, B. V. Smith, and V. V. Yashchuk, "Performance of the upgraded LTP-II at the ALS Optical Metrology Laboratory," Proc. SPIE 7077, 7077-10/112 (2008).

[6] http://www.moeller-wedel-optical.com/El-Autocolimators/G_Elcomat3000.htm

[7] R.D. Geckeler, "Optimal use of pentaprisms in highly accurate deflectometric scanning," Measurement and Science Technology 18 (2007) 115-125.

[8] V. V. Yashchuk, "Optimal Measurement Strategies for Effective Suppression of Drift Errors," (submitted to Rev. Sci. Instrum.).

[9] V. V. Yashchuk, W. R. McKinney, T. Warwick, T. Noll, F. Siewert, T. Zeschke, and R. D. Geckeler, "Proposal for a Universal Test Mirror for Characterization of Slope Measuring Instruments," Proc. SPIE 6704, 67040A/1-12 (2007). 